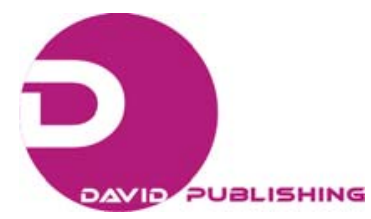

\title{
Characterization and Synthesis of Carbon Nanotubes
}

\author{
Afaf Ali Gadalla and Mohanned Mohamed Mahmod \\ Physics Department, Faculty of Science, Assiut University, Assiut 71516, Egypt
}

Received: June 02, 2010 / Accepted: July 06, 2010 / Published: February 10, 2011.

\begin{abstract}
The discovery of carbon nanomaterials has added to the applications of carbon and created enormous interest in their development, characterization and applications in many different fields. This study describes the production and properties of carbon nanotubes (CNT), with the aim of drawing attention to useful available information and to enhancing interest in this new highly advanced technological field for researcher and the manufacturing engineer. Single wall carbon nanotubes (SWNTs) were synthesized using DC arc discharge reactor. Then an efficient purification procedure which combines centrifugation and precipitation has been used. As shown in Scanning Electron Microscopy (SEM) micrographs the cluster of metals turns smaller and smaller and the bundles of the carbon nanotube appear more and more gradually depending on the degree of purification. The thermal behavior and stability of SWCNTs-Water has been investigated by Differential Scanning Calorimeter (DSC). Also, X-ray diffraction (XRD) of SWNT for different $\mathrm{Y}$ and Ni catalyst has been characterized by powder X-ray diffraction.
\end{abstract}

Key words: Single wall carbon nanotubes, degree of purification, scanning electron microscopy, X-ray diffraction.

\section{Introduction}

Single wall carbon nanotubes (SWNTs) have attracted scientific interest because of their fascinating physical properties and for their application in materials physics and the chemical industry. This application includes modern electronic devices [1, 2], molecular storage like hydrogen storage [3], field emission displays [4], transistors [5], electrochemical devices such as actuators [6], supercapacitors or charge storage [7], nanocomposites, sensors [8], nanometersized semiconductor devices, interconnects [9], conductive high-strength composites, nanofluidic devices [10] and one-dimensional cellular probes [11]. Nanotube samples of higher purity are strongly needed for many research and application purposes. Many scientists have been theoretically trying to search the structure of confined water in carbon nanotubes [12]. Water in restricted spaces is expected to give unusual water behavior in interior of nanotube as well as in the grooves between adjacent nanotubes on the outside

Corresponding author: Afaf Ali Gadalla (1943- ), female, professor, research fields: materials science and nanophysics. E-mail: Afaf_gadalla1@yahoo.com. surface of SWCNT bundle, the behavior should adjacent differ from that of bulk water [13]. Oleg Byl [14] presented in his study by using vibration spectroscopy evidence of a water phase inside single walled carbon nanotubes that exhibits an unusual form of hydrogen-bonding at temperature between 123 and 183 K. After the discovery of carbon nanotubes, Ebbesen and Ajayan [2] successfully have achieved a large-scale synthesis which made extensive investigation on this promising material possible.

\section{Experiment}

\subsection{SWCNTs Sample Preparation}

Some of the SWNTs (NiY) samples are produced by using the method which has been reported by Journet [15]. Briefly, the SWNTs are synthesised using the DC arc discharge reactor. The product of arc-discharge synthesis is in a powder form that includes, carbon nanotubes, amorphous carbon and metal catalyst particles (Ni, Y and Fe clusters) which are generally coated with a layer of carbon in amorphous form and carbon nanocapsules (bucky-onions). SWCTs have to 
separate from the other parts. Synthesis and extraction process to obtain samples of SWCNTs is as follows:

A graphitic rod containing a mix of carbon and metal catalyst powder is used as an anode in the reactor of nanotubes. Moreover, a hole (3.2 mm diameter, 8.30 $\mathrm{mm}$ depth) is drilled in graphite rod which is filled with a mix of carbon and metal catalyst powder, Y (7\% atomic weight), Ni (28 atomic weight) and C (65\% atomic weight). Then this rod is loaded into the reactor and acts as the consumed anode for the process. The cathode is a large plate made from high density carbon and is not consumed in the process. The chamber is sealed and pumped down at 500 mbar. A direct current (d. c.) source is connected between the crocodile pliers is plugged in and set to provide 100 amperes at a voltage of 60 volts. The production is initiated by adjusting the distance between the anode and the cathode maintaining the voltage constant $(60 \mathrm{~V})$. The nanotubes start to grow below $1100{ }^{\circ} \mathrm{C}$. A bright discharge occurs and the anode is consumed. The electrode separation of about $1 \mathrm{~mm}$ must be maintained by the operator for the duration of the process which can take about six minutes and then the electrical supply may be automatically switched off. The chamber has to be left cool down by filling it with Helium; finally the reactor will be opened slowly. After finishing the process, a raw material (CNT) was collected from the place with the highest and lowest concentration of nanotubes and characterized by the XRD to prove that there are nanotubes produced in the batch. This gives an indication of the success of the production process. Also, the energy-dispersive X-ray microanalysis (EDX) diagram of heated SWCNT was done.

\subsection{Oxidation in Air Purification}

There are many purification techniques, each of them has its own advantages and limitations. For instance, chemical oxidation treatments are conveniently used in large batch production, but almost they destroy carbon nanotubes layers [16].
After finishing the raw material production and seeing that there are carbon nanotubes in the batch, then all parts of product have to be collected from the outside edges of cathode, chamber walls and web deposits. The different types of the batch are put in the oven in air by $350{ }^{\circ} \mathrm{C}$ for three hours or until $25 \%$ of its weight has been lost. Most of the amorphous carbon will be removed by this process.

\subsection{Acidic Purification Treatments of SWCNT}

The oxidation procedure is used to remove the metal impurities through opening the carbon shell, where the metal clusters are encapsulated and removing the other graphitised carbon structures. It also opens the closed nanotubes. Most of purification methods employ acid treatment to dissolve the metal particles, whereas air oxidation is applied to remove the amorphous carbon [8]. Therefore, it is important to use an easy and convenient process to obtain pure samples of SWCNTs. An efficient purification procedure which combines centrifugation and precipitation has been used. The process includes three main steps: (1) The raw soot is treated in $65 \%$ nitric acid for three hours. During this time a weight loss of about $20 \%$ occurs. After the black solution is centrifuged, black sediment remains at the bottom of the centrifuge tube. The clear brownish-yellow supernatant acid is decanted off. The sediment still contains substantial amounts of trapped acid. Thereafter the supernatant liquid has to be centrifuged and decanted. After these successive washing-centrifugation cycles, the liquid phase becomes less acidic. The remaining solution which is left behind after a mixture is centrifuged (supernatant) and it becomes firstly colourless and then increasingly dark. (2) The remaining sediment is dispersed in deionised water by treating it with ultrasonic pulses for one minute. (3) After finishing the acidic treatment, high temperature ( $350^{\circ} \mathrm{C}$ for one hour) heating of the purified samples has been adopted to remove the chemical functional groups, created on the nanotube surface due to acid treatment. 


\section{Results and Discussion}

\subsection{The Energy-Dispersive X-Ray Microanalysis Diagram (EDX)}

Fig. 1 shows the energy-dispersive X-ray microanalysis diagram (EDX) of heated SWCNT $\left(350^{\circ} \mathrm{C}, 12 \mathrm{~h}\right.$ ) versus the concentration of elements in the sample in counts per second (cps). It demonstrates different elements in the sample $(\mathrm{Ni}=4.55, \mathrm{Y}=2.43$ weight $\%$ from the entire sample).

\subsection{Scanning Electron Microscopy (SEM)}

Fig. 2 shows a scanning electron microscopy (SEM) image of a raw material containing bundle of nanotubes, amorphous carbon and cluster of Ni, Y, while Fig. 3 shows SWCNT material with more bundles of carbon nanotubes as a result of more purification.

As shown in the previous figures, the cluster of metals turns smaller and smaller, the bundles of carbon nanotubes appear more and more gradually depending on the degree of the purification.

\subsection{Differential Scanning Calorimetery}

The thermal behaviour and stability of SWCNTs-water have been investigated by differential scanning calorimetery (DSC). The results represented in Fig. 4 show the behaviour of water in opened SWCNT, which contains water adsorbed on SWCNTs. The first peak from SWCNTs loaded $100 \%$ of water appeared at $103.6{ }^{\circ} \mathrm{C}$ and represents only $6.7 \%$ of the total area, where the second one at $132.1{ }^{\circ} \mathrm{C}$ and represents $93.3 \%$, that means the great part of water is not bulk water behaves unusually. The unusual behaviour may relate to the strong hydrogen bonding of water which exists inside the nanotubes.

\subsection{X-Ray Diffraction}

Fig. 5 shows the XRD diffractograms of SWNT for different Ni Y characterized at each stage by powder $\mathrm{X}$-ray diffraction. The curves a, b and c in Fig. 5 belong to raw SWCNTs, heat treated by $370{ }^{\circ} \mathrm{C}$ and four times

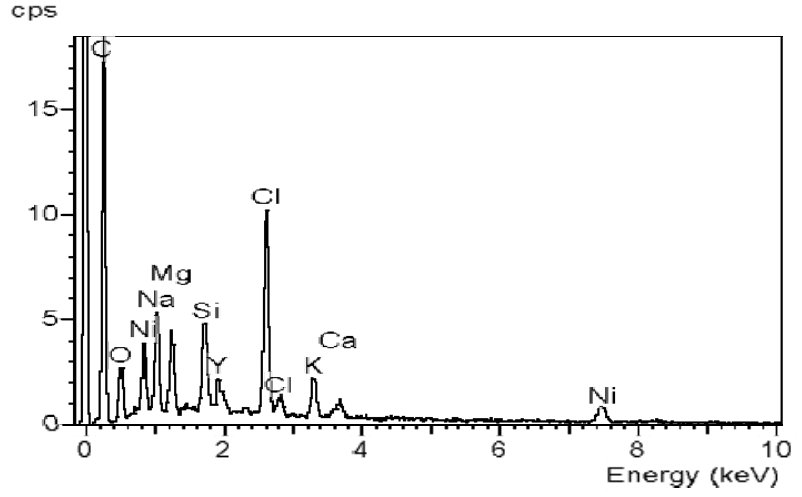

Fig. 1 The EDX diagram of cluster metals used in generation of carbon nanotubes ( $\mathrm{NiY})$.

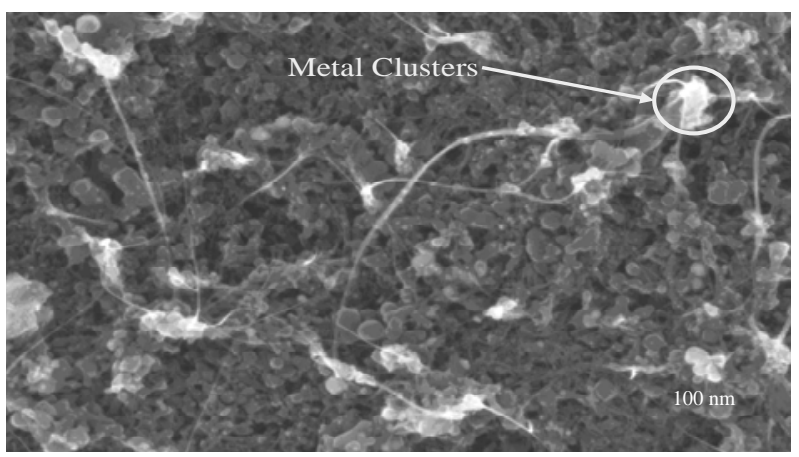

Fig. 2 SEM micrograph of raw SWCNTs (Ni Y).

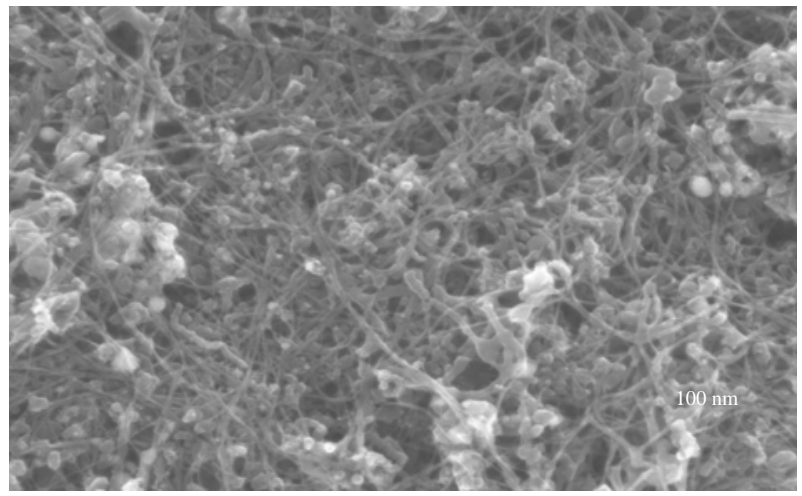

Fig. 3 SEM micrograph of SWCNTs (Ni Y) after four cycle of purification.

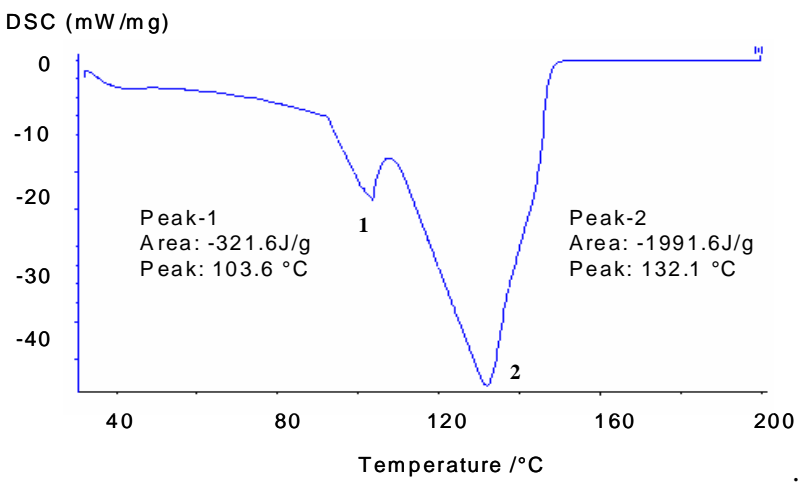

Fig. 4 The thermal behaviour of opened SWCNT. 


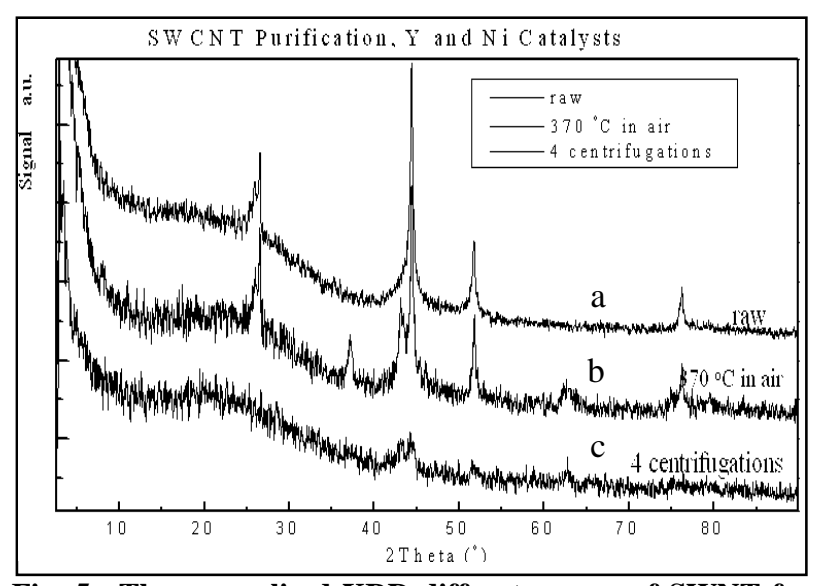

Fig. 5 The normalised XRD diffractograms of SWNT for different NiY characterized at each stage by powder $\mathrm{X}$-ray diffraction.

centrifuged samples, respectively. The diffraction peaks, where the lines are indicated, correspond to the three most intense peaks of Ni Y carbide. Those peaks are calculated at $37^{\circ}, 45^{\circ}$ and $52^{\circ}$, respectively. The peak at $\sim 43^{\circ}$ corresponds to the diffraction peak of graphite and is also present in nanotubes. The more intense carbide peaks could be due to carbide inside SWNT whose diameter is bigger for high iron ratios. Lower size of the NiY particles serves as nucleation centres for the nanotube growth.

\section{Conclusions}

Single-wall nanotube samples have been produced with sufficient quantities to determine its structure. Further enhancement of the carbon nanotube growth technique raised the nanotube yields. The success of the production was proved by X-ray diffraction process. An efficient purification procedure has been used which combines centrifugation and precipitation process. The present scanning electron micrographs showed that more bundles of carbon nanotube have appeared depending on the degree of purification. The results of the thermal analysis indicates that SWCNT have two types of observed water one inside and the other outside that means great part of water is not bulk water but behaves unusually. The XRD analysis of SWNT for different $\mathrm{Y}$ and Ni catalysis indicates that the small nanoparticles encapsulated inside the nanotube which cannot be removed with different purification methods.

\section{References}

[1] S. Iijima, Helical microtubules of graphitic carbon, Nature 364 (1991) 56-58.

[2] T.W. Ebbesen, P.M. Ajayan, Large scale synthesis of carbon nanotubes, Nature (London) 358 (1992) 220-222

[3] R. Bacon, Growth, structure, and properties of graphite whiskers, Appl. Phys. 31 (1960) 283-285.

[4] W. Kratchemer, L.D. Lamb, K. Fostiropoulos, D.R. Hufffman, Solid C60 : a new form of carbon, Nature 37 (1990) 354-358.

[5] P.A. Ajayan, S. Iijima, Carbon fiber method for their production, Nature 358 (1992) 23-27.

[6] V.P. Dravid, X. Lin, Y. Wang, X.K. Wng, A. Yee, J.B. kettrson, R.P.H. Chang, Carbon nanotubes synthesized in hydrogen arc discharg and derivaties their growth and implication for buckyball formation, Science 259 (1993) 1601-1604.

[7] S. Iijima, Chirality correlation in double-wall carbon nanotubes as studied by electron diffraction, Mater. Sci. Eng. B 19 (1993) 173-176.

[8] A.G. Rinzeler, Luiu, H. Dai, P. Nikolaev, C.B. Huffman, Large scale purification of single wall carbon nanotubes: process, product and characterization, Appl. Phys. A 67 (1998) 29-33.

[9] A. Thess, R. Lee, P. Nikolaev, H. Dai, J. Robert, Metallic resistivity in crystalline ropes of single-wall carbon nanotubes, Science 273 (1996) 483-487.

[10] W.S. Eisebitt, G. Kann, W. Eberhardt, Nanotubes, CRC, Boca Raton, Frontiers of nano-optoelectronic systems, Phys. Rev. B 61 (2000) 5719-5725.

[11] J. Kong, J.H.M. Cassell, C.F. Quate, H. Dai, Functionalized carbon nanotubes for nuclear hydrogen sensors, Nature 395 (1998) 878-883.

[12] T. Meng-Hung, L. Hong-Min, T. Wen-Li, H. Yeukuang, Examine ths GAS absorption properties of single wall carbon nanotube bondes by X-ray absorption techniques, Rev. Adv. Mater. Sci.5 (2003) 302-305.

[13] A.I. Kolesnikov, J.M. Zanotti, C.K. Loong, P. Thiyagarajan, A.P. Moravsky, R.O. Loutfy, C.J. Burnham, Diffusion of single alkaline molecule in carbon nanotube studied by molecular dynamic simulation, Phys. Rev. Lett. 93 (2004) 035503.

[14] B. Oleg, L. Jin-Chen, W. Yang, Y. Wai-Leung, J. Karl, Unusual hydrogen bonding in water-filled carbon nanotubes, JACS, 2006, available online at: www.biomedexperts.com/Profile.bme/178082.

[15] C. Journet, M.K. Maser, P. Bernier, A. Loiseau, M. Lamy de la Chapelle, S. Lefrant, P. Deniard, R. Lee, J.E. Fischer, Large scale production of single wall carbon nanotubes by the electric arc technique, Nature (London) 388 (1997) 756-758.

[16] T.W. Ebbesen, Carbon Nanotubes: Preparation and Properties, CRC Press, New York, 1999, p. 45. 